Attention deficit hyperactivity disorder (ADHD) is a common neurodevelopmental disorder, characterised by a persistent pattern of inattention and/or hyperactivity-impulsivity, directly impacting on academic, occupational, or social functioning. It affects between $1-5 \%$ of children and young people (CYP) most often presenting in early-mid childhood.

Pharmacological treatment can be considered in CYP if certain criteria are met, where licensed medications include methylphenidate, dexamfetamine, lisdexamfetamine, atomoxetine and guanfacine. Stimulant and non-stimulant medications require frequent physical health monitoring due to their side effects including an increase in blood pressure and/or heart rate, loss of appetite, growth restriction and tics.

Method. Standards and criteria were derived from the NICE guidance (2018), whilst local trust policies were reviewed, demonstrating discrepancies. Standards were expected to be met for $100 \%$ of patients.

Electronic patient records were reviewed retrospectively from a representative cohort of CYP reviewed by clinicians in a community CAMHS service during March-November 2020. Data were entered manually into a spreadsheet for evaluation.

Result. A total of 27 CYP records were reviewed, average age 13 yo, on a range of stimulant/non-stimulant preparations.

5 (19\%) had height checked every 6 months, with 4 delayed to 7-8 months.

For those >10yo, only 5 (19\%) had weight checked every 6 months.

Only $2(7 \%)$ had their height and weight plotted on a growth chart and reviewed by the healthcare professional responsible for treatment.

Just 4 (15\%) had heart rate and blood pressure recorded before and after each dose change, whilst similarly only 4 (not the same) had these parameters recorded every 6 months.

17 patients were reviewed by telephone/video call, where 5 patients provided physical health parameters (measured at home). Conclusion. Across all parameters, standards are not being met for the required physical health monitoring for CYP on ADHD medication.

The COVID-19 pandemic has significantly changed the working conditions for community teams, impacting face to face reviews, creating challenges for physical health monitoring.

Our ongoing implementations for change include the use of a proforma for physical health measurements, improving psychoeducation for families, exploring potential barriers with senior colleagues and collaborating with pharmacy colleagues to update local guidelines in accordance with the latest NICE recommendations. We aim to re-audit in June 2021.

\section{Are the staff in Heddfan Psychiatric Unit, Wrexham Maelor Hospital, are adhering to the personal protective equipment (PPE) guidance as per Public Health Education, England? a QIP}

Asha Dhandapani*, Sathyan Soundararajan, Rajvinder Sambhi and Catherine Baker

\section{BCUHB}

${ }^{\star}$ Corresponding author.

doi: 10.1192/bjo.2021.498

Aims. The aim of this audit is to assess whether healthcare staff are correctly donning and doffing PPE when entering and leaving the wards (changed to donning and doffing PPE when within 2 metres vicinity of a patient).
Method. Consultants/ Junior doctors/ Ward managers/ Staff nurses/ student nurses/ Health care support workers/ Occupational therapist/ Psychologists/ Student nurses/ Housekeeping staff, were all included in this Audit. None of the staff was aware of this Audit and this was an entirely random observation. We used a standard proforma in order to audit. Followed by the Audit, we trained the staff in the unit and then re-audited.

Result. $98 \%$ of them wore mask whilst in the ward and $94 \%$ of them washed their hands after doffing. 36\% did not wear them appropriately and about $10-14 \%$ did not wear PPE at all. A mere 7 out of 50 alone used hand gel. Overall the donning and doffing of PPE was not being followed and adhered to according to the standards from PHE as per the first Audit. In particular, during donning only 1/3rd of them donned the PPE as per guidance. Likewise, the doffing technique was also poor, with only half of them removing the apron and mask correctly. Unfortunately, only 7 of the 50 people were observed to have used hand gel in between the doffing. This could be potentially increasing the risk of the spread of the coronavirus.

We had trained almost 150 staff members in the Heddfan unit with regard to $\mathrm{PPE} /$ donning and doffing.

Handwashing prior to donning was achieved by all the staff. All the staff, that is $100 \%$ of them adhered to the donning technique in line with the guidance in comparison to just $64 \%$ during the first Audit. Whilst hardly just $1 / 2$ to $2 / 3$ rd of the staff followed the doffing technique adequately, the second audit showed that only 2 of the 50 staff did not follow the guidance. A meagre/ handful of them followed the utilisation of hand gel in between the tasks of doffing during the first Audit. Almost $90 \%$ of them followed the technique properly during the second Audit. Thus showing that the PPE training was successful.

Conclusion. Following the PPE training that was provided to them there was a good response from the staff and this went on to show how effectively we have managed the prevention/ contamination of virus in our unit.

Are the rapid tranquilisation nice guidelines adhered to, in patients with agitated/aggressive behavior? a QIP

Asha Dhandapani*, Sathyan Soundararajan

and Rajvinder Sambhi

BCUHB

${ }^{\star}$ Corresponding author.

doi: 10.1192/bjo.2021.499

Aims. To explore whether the NICE guidelines for rapid tranquilisation are adhered to in the Psychiatric intensive care unit (PICU/ Tryweryn).

Method. Data were collected by core trainees. Standards were taken from NICE guidelines NG10. All patients who had received rapid tranquilisation, that were in PICU from August 2019 to February 2020 were considered in this a.

Result. During the first PDSA, we discussed with the staff in the ward regarding the protocol. Prior to actually starting the second audit, the adherence was noted to be low. However following persistence and having created a protocol jointly with the ward manager, we could see the difference. The staff were appreciated for their efforts in maintaining $100 \%$ adherence. The same was intended to be continued with some positive reinforcement from the auditing team. Over the first 2 months, 12 patients received Rapid Tranquilisation. Out of these 12, we randomly selected 4 patients to find the adherence of the NICE guidelines to be 100 per cent. The predictions regarding the adherence to protocol showed that the PDSA was successful.

During the second PDSA, the adherence was 100\% again. The adherence to the protocol has been followed for not just the 